\title{
Higgs form factors in associated production
}

\author{
Gino Isidori ${ }^{a, b}$ and Michael $\operatorname{Trott}^{a}$ \\ a Theory Division, Physics Department, CERN, \\ CH-1211 Geneva 23, Switzerland \\ ${ }^{b}$ INFN, Laboratori Nazionali di Frascati, \\ I-00044 Frascati, Italy \\ E-mail: gino.isidori@lnf.infn.it, michael.trott@cern.ch
}

ABSTRACT: We further develop a form factor formalism characterizing anomalous interactions of the Higgs-like boson $(h)$ to massive electroweak vector bosons $(V)$ and generic bilinear fermion states $(\mathcal{F})$. Employing this approach, we examine the sensitivity of $p p \rightarrow \mathcal{F} \rightarrow V h$ associated production to physics beyond the Standard Model, and compare it to the corresponding sensitivity of $h \rightarrow V \mathcal{F}$ decays. We discuss how determining the $V h$ invariant-mass distribution in associated production at LHC is a key ingredient for model-independent determinations of $h V \mathcal{F}$ interactions. We also provide a general discussion about the power counting of the form factor's momentum dependence in a generic effective field theory approach, analyzing in particular how effective theories based on a linear and non-linear realization of the $\mathrm{SU}(2)_{\mathrm{L}} \times \mathrm{U}(1)_{\mathrm{Y}}$ gauge symmetry map into the form factor formalism. We point out how measurements of the differential spectra characterizing $h \rightarrow V \mathcal{F}$ decays and $p p \rightarrow \mathcal{F} \rightarrow V h$ associated production could be the leading indication of the presence of a nonlinear realization of the $\mathrm{SU}(2)_{\mathrm{L}} \times \mathrm{U}(1)_{\mathrm{Y}}$ gauge symmetry.

Keywords: Higgs Physics, Beyond Standard Model

ARXIV EPRINT: 1307.4051 


\section{Contents}

1 Introduction 1

2 Amplitude decomposition 3

2.1 Generalization to arbitrary currents 4

3 General EFT power counting for the form factors 5

3.1 General decomposition 5

$\begin{array}{lll}3.2 & \text { Constant terms } & 6\end{array}$

3.3 Slopes in $q^{2}$ from contact interactions $\quad 6$

$\begin{array}{ll}3.4 & \text { The photon pole }\end{array}$

$\begin{array}{lll}3.5 \text { Summary } & 7\end{array}$

4 The form factors in explicit EFTs $\quad 8$

$4.1 \quad$ Non-Linear realization of $\mathrm{SU}(2)_{\mathrm{L}} \times \mathrm{U}(1)_{\mathrm{Y}} \quad 9$

4.2 Linear realization of $\mathrm{SU}(2)_{\mathrm{L}} \times \mathrm{U}(1)_{\mathrm{Y}} \quad 11$

4.3 Linear vs. Nonlinear realization of $\mathrm{SU}(2)_{\mathrm{L}} \times \mathrm{U}(1)_{\mathrm{Y}} \quad 12$

5 Associated production as a probe of anomalous couplings 12

$\begin{array}{lll}5.1 & \text { Current data } & 12\end{array}$

$\begin{array}{lll}5.2 & \text { Future associated production spectra } & 14\end{array}$

$\begin{array}{ll}5.3 & \text { Breit-Wigner smearing and experimental reconstruction uncertainty } \quad 15\end{array}$

$\begin{array}{lll}5.4 & \text { PDF effects, further distributions } & 16\end{array}$

6 Conclusions 18

\section{Introduction}

In this paper we study the utility of scalar production in association with a single massive electroweak vector boson $\left(W^{ \pm}\right.$or $Z^{0}$, generically denoted by $V$ ) in constraining physics beyond the Standard Model (SM), and determining if the observed Higgs-like Boson is precisely the SM Higgs Boson. Further developing the formalism introduced in ref. [1], we characterize the interactions of the Higgs-like boson, $h$, to $V$ and a generic bilinear fermion state $|\mathcal{F}\rangle=|\bar{\psi} \psi\rangle$ by means of a set of form factors, depending only on the $\mathcal{F}$ invariant mass $\left(q^{2}\right)$. This formalism has a twofold advantage. On the one hand it is very general: the information about physics beyond the SM that can be extracted from processes of the type $h \rightarrow V \mathcal{F}$ (or $p p \rightarrow \mathcal{F} \rightarrow h V$ ) can be encoded in the $h V \mathcal{F}$ form factors, provided the creation (or annihilation) of the state $\mathcal{F}$ is described by a local bilinear fermion current. On the other hand, the formalism allows us to relate different physical processes based on the same fundamental three-point $h V \mathcal{F}$ Green's function. In particular, it allows us to 
compare, under a minimal set of assumptions, the sensitivity to physics beyond the SM (BSM) of $\mathcal{F} \rightarrow V h$ associated production to that of $h \rightarrow V \mathcal{F}$ decays.

The production of $h V$ at large invariant mass is potentially accessible at the LHC and offers a key probe of the $h V \mathcal{F}$ form factor behavior at large $q^{2}$. Probing for the large $q^{2}$ behavior of the three-point $h V \mathcal{F}$ Green's function is not possible by looking at $h \rightarrow V F$ decays only [1], which are restricted to low $q^{2}$. We show how this information can be extracted from future LHC data by measuring the appropriate differential distributions and then mapping it onto an underlying EFT formalism.

The extra sensitivity to BSM effects in the reconstructed differential distributions follows from the introduction of non-standard $q^{2}$ dependence in the form factors. In this respect, our decomposition into $h V \mathcal{F}$ form factors is a more detailed study than past analyses of the $h \rightarrow V V^{*}$ amplitude [2-5]. While these previous works have analyzed in generality the possible Lorentz-structure of the $h \rightarrow V V^{*}$ amplitude, even assuming nonstandard spin and parity properties for $h$, they have frequently implicitly assumed that the $h \rightarrow V V^{*}$ amplitude can be unambiguously determined by the structure of an (hypothetical) on-shell $h V V$ vertex, or if this assumption was not made, they have practically neglected the $q^{2}$ dependence of the form factor in detailed analyses. Since the $h V V$ interaction is not kinematically accessible given the experimental values of $m_{h}$ and $V$, it is important to include the effects of non-standard $h V \mathcal{F}$ contact interactions. ${ }^{1}$

Generalizing the analysis of ref. [1], in this work we develop the decomposition of the $h V \mathcal{F}$ amplitude in the presence of generic contact interactions, whose effect can be incorporated in the form-factor formalism via non-SM $q^{2}$ dependence. We also analyze the power counting of the form factor's momentum dependence in a generic effective field theory (EFT) approach. Given the experimental evidence of a Higgs-like boson, and the absence of non-SM particles at the LHC, two effective theories are of particular current interest: an EFT with a non-linear realization of $\mathrm{SU}(2)_{\mathrm{L}} \times \mathrm{U}(1)_{\mathrm{Y}}$, where $h$ is introduced as an $\mathrm{SU}(2)_{\mathrm{L}} \times \mathrm{U}(1)_{\mathrm{Y}}$ scalar singlet, and the EFT based on the linear realization of the $\mathrm{SM}$ gauge group, where $h$ belongs to an $\mathrm{SU}(2)_{\mathrm{L}}$ doublet. We show how these two EFT's map into the form factors. As a by-product, we clarify how possible deviations from the SM in the $h V \mathcal{F}$ differential spectra could provide a clear clue to distinguish between these two EFT approaches. The mapping into an underlying EFT is in fact required to evaluate the form factors from the theoretical point of view. However, this fact does not diminish the usefulness of the form factor formalism as an intermediate analysis step in a decomposition of the $h \rightarrow V \mathcal{F}, \mathcal{F} \rightarrow h V$ amplitudes that allows us to potentially distinguish the two EFT approaches, as we show.

In other words, the form-factor approach provides us a compact and very general tool to map possible experimental measurements into information on some underlying field theory, minimizing the assumptions on the latter (for instance, without specifying of how $\mathrm{SU}(2)_{\mathrm{L}} \times \mathrm{U}(1)_{\mathrm{Y}}$ is realized in the scalar sector). Of course one must map onto an explicit EFT to perform perturbative corrections and define the amplitude beyond leading order.

\footnotetext{
${ }^{1}$ Previous studies on associated production of the Higgs-like Boson include ref. [6-17]. See also ref. [1822] for some other recent analyses in the EFT context.
} 
However, one of the purposes of this paper is to make clear the manifest utility, and the technical simplification, that using form factors as an intermediate step in the calculation (and in data analysis) supplies.

The outline of the paper is as follows. In the next section we introduce the $h V \mathcal{F}$ form factors and discuss how they modify of the SM cross section formulae for associated Higgs production. The general power counting decomposition of the the form factors is presented in section 3, and their explicit evaluation within the linear and nonlinear EFTs is discussed in section 4. A numerical study of BSM effects in associated production is presented in section 5. The results are summarized in section 6 .

\section{Amplitude decomposition}

Following ref. [1], we start with the general decomposition of the $h \rightarrow V \mathcal{F}$ transition amplitude, where $V$ is an (approximately) on-shell massive weak gauge boson, and $\mathcal{F}$ is a state generated at tree level by the electroweak charged or neutral currents, $J_{\mu}^{V}$, defined by

$$
\mathcal{L}_{J}^{\mathrm{SM}}=\frac{e}{\sqrt{2} \sin \theta_{W}} J_{\mu}^{ \pm} W_{ \pm}^{\mu}+\frac{e}{\sin \theta_{W} \cos \theta_{W}} J_{\mu}^{0} Z^{\mu}=\sum_{V} C_{V} g_{V} J_{\mu}^{V} V^{\mu}
$$

Here $g_{V}=\left\{g_{2}, g_{2} / \cos \theta_{W}\right\}, g_{2}=e / \sin \theta_{W}$, and $C_{V}=\{1 / \sqrt{2}, 1\}$ are the coupling and normalization factors for $V=\left\{W^{ \pm}, Z^{0}\right\}$. Defining $J_{\mu}^{\mathcal{F}_{V}}=\left\langle\mathcal{F}\left|J_{\mu}^{V}\right| 0\right\rangle$, the transition amplitude can be decomposed in terms of four independent Lorentz structures

$$
\begin{aligned}
\mathcal{A}_{V}^{\mathcal{F}} & =\mathcal{A}[h \rightarrow V(\tilde{\epsilon}, p) \mathcal{F}(q)]=\frac{C_{V} g_{V}^{2} m_{V}}{\left(q^{2}-m_{V}^{2}\right)} \tilde{\epsilon}_{\mu} J_{\nu}^{\mathcal{F}_{V}} T_{V}^{\mu \nu}, \\
T_{V}^{\mu \nu} & =\left[f_{1}^{V}\left(q^{2}\right) g^{\mu \nu}+f_{2}^{V}\left(q^{2}\right) q^{\mu} q^{\nu}+f_{3}^{V}\left(q^{2}\right)\left(p \cdot q g^{\mu \nu}-q^{\mu} p^{\nu}\right)+f_{4}^{V}\left(q^{2}\right) \epsilon^{\mu \nu \rho \sigma} p_{\rho} q_{\sigma}\right] .
\end{aligned}
$$

The amplitude in eq. (2.2) controls both the $h \rightarrow V \mathcal{F}$ decay and the $V h$ associated production process, $\mathcal{F} \rightarrow V h$. Denoting $q$ the total momentum of the initial state in the partonic process $\bar{\psi} \psi \rightarrow V h$, we can write

$$
\mathcal{A}[\bar{\psi} \psi(q) \rightarrow h V(\tilde{\epsilon},-p)]=\mathcal{A}_{V}^{\psi \bar{\psi}}
$$

where $\mathcal{A}_{V}^{\bar{\psi} \psi}$ is decomposed exactly as in eq. (2.2). The important difference between $h$ decays and $V h$ associated production is the allowed kinematical region probed by the process: $0<q^{2}<\left(m_{h}-m_{V}\right)^{2}$ in $h$ decays, and $q^{2}>\left(m_{h}+m_{V}\right)^{2}$ in the $V h$ associated production. This fact can lead to an enhanced sensitivity to BSM effects in kinematic distributions of associated production compared to kinematic distributions of $h \rightarrow V \mathcal{F}$ decays.

The partonic cross sections for the $V h$ associated production within the SM, at fixed $q^{2}$, are well known [23]

$$
\begin{aligned}
\sigma(\bar{\psi} \psi \rightarrow Z h)^{\mathrm{SM}} & =\sigma_{0}^{Z h} \frac{\left|\vec{p}_{h}\right|}{\sqrt{q^{2}}} \frac{\left|\overrightarrow{p_{h}}\right|^{2}+3 m_{Z}^{2}}{\left(q^{2}-m_{Z}^{2}\right)^{2}}, & \sigma_{0}^{Z h} & =\frac{2 \pi \alpha^{2}\left[\left(g_{\psi}^{L}\right)^{2}+\left(g_{\psi}^{R}\right)^{2}\right]}{12 N_{c} \sin ^{4} \theta_{W} \cos ^{4} \theta_{W}}, \\
\sigma\left(\bar{\psi}_{j} \psi_{i} \rightarrow W h\right)^{\mathrm{SM}} & =\sigma_{0, i j}^{W H} \frac{\left|\overrightarrow{p_{h}}\right|}{\sqrt{q^{2}}} \frac{\left|\overrightarrow{p_{h}}\right|^{2}+3 m_{W}^{2}}{\left(q^{2}-m_{W}^{2}\right)^{2}}, & \sigma_{0, i j}^{W h} & =\frac{\pi \alpha^{2}\left|V_{i j}\right|^{2}}{18 \sin ^{4} \theta_{W}},
\end{aligned}
$$


where $\left|\overrightarrow{p_{h}}\right|=\left[\left(q^{4}+m_{h}^{4}+m_{V}^{4}-2 q^{2} m_{h}^{2}-2 q^{2} m_{V}^{2}-2 m_{V}^{2} m_{h}^{2}\right) /\left(4 q^{2}\right)\right]^{1 / 2}$ is the center of mass momentum of the Higgs-like boson and $V_{i j}$ denotes CKM matrix elements. ${ }^{2}$ Here

$$
g_{\psi}^{L}=2 T_{3}^{\psi}-2 Q_{\psi} \sin ^{2} \theta_{W}, \quad g_{\psi}^{R}=-2 Q_{\psi} \sin ^{2} \theta_{W},
$$

are the left-handed and right-handed coupling of the $Z$ boson, normalized as in eq. (2.1). Using the SM rate as a normalization, the generalized partonic results in the case of the form factor decomposition in eq. (2.2) assumes the following simple form

$$
\begin{aligned}
\frac{\sigma^{\mathrm{BSM}}(\psi \bar{\psi} \rightarrow V h)}{\sigma^{\mathrm{SM}}(\psi \bar{\psi} \rightarrow V h)} \equiv & R_{V}\left(q^{2}\right)=\left|f_{1}^{V}\left(q^{2}\right)\right|^{2}+3 \operatorname{Re}\left[f_{1}^{V}\left(q^{2}\right) f_{3}^{V *}\left(q^{2}\right)\right] \frac{m_{V}^{2}\left(q^{2}+m_{V}^{2}-m_{h}^{2}\right)}{\left|\overrightarrow{p_{h}}\right|^{2}+3 m_{V}^{2}} \\
& +\frac{m_{V}^{2} q^{2}}{\left|\overrightarrow{p_{h}}\right|^{2}+3 m_{V}^{2}}\left[\left|f_{3}^{V}\left(q^{2}\right)\right|^{2}\left(3 m_{V}^{2}+2\left|\overrightarrow{p_{h}}\right|^{2}\right)+2\left|\overrightarrow{p_{h}}\right|^{2}\left|f_{4}^{V}\left(q^{2}\right)\right|\right] .
\end{aligned}
$$

\subsection{Generalization to arbitrary currents}

In writing eq. (2.2) we have assumed that the state $\mathcal{F}$ is generated only by the weak currents in eq. (2.1). This assumption is motivated by the fact that this is the only allowed tree-level contribution to the $h \rightarrow V \mathcal{F}$ amplitude within the SM. With this assumption, the decomposition in eq. (2.1) is aimed at characterizing anomalous Higgs interactions with the $\mathrm{SU}(2)_{\mathrm{L}} \times \mathrm{U}(1)_{\mathrm{Y}}$ gauge bosons.

Nevertheless, this assumption is not necessarily a good approximation in generic extensions of the SM. The decomposition in eq. (2.2) can be easily generalized to all cases where $\mathcal{F}$ is generated by generic local left-handed or right-handed fermion current,

$$
\left(J_{L}^{i j}\right)_{\mu}=\bar{\psi}_{L}^{i} \gamma_{\mu} \psi_{L}^{j} \quad \text { or } \quad\left(J_{R}^{i j}\right)_{\mu}=\bar{\psi}_{R}^{i} \gamma_{\mu} \psi_{R}^{j},
$$

provided we enlarge the number of independent form factors. In general, for each independent current $J$ contributing to the final state $\mathcal{F}$, we have a set of four independent form factors $\left(f_{1-4}^{V_{J}}\right)$ associated with the four different Lorentz structures appearing in eq. (2.2). In the limit of a $\mathrm{SU}(3)^{5}$ unbroken flavour symmetry, there are five independent currents $\left(J^{f}\right)_{\mu}=\bar{f} \gamma_{\mu} f$, where $f=\left\{u_{R}, d_{R}, e_{R}, L_{L}, Q_{L}\right\}$, and at most two of them $\left(J_{L}^{\mathcal{F}}, J_{R}^{\mathcal{F}}\right)$ can create or annihilate the state $\mathcal{F}$ at the tree level. The trivial generalization of eq. (2.2) then reads

$$
\mathcal{A}_{V}^{\mathcal{F}}=\frac{C_{V} g_{V}^{2} m_{V}}{\left(q^{2}-m_{V}^{2}\right)} \sum_{J=J_{L}^{\mathcal{F}}, J_{R}^{\mathcal{F}}} \tilde{\epsilon}_{\mu}\left\langle\mathcal{F}\left|J_{\nu}\right| 0\right\rangle T_{V_{J}}^{\mu \nu},
$$

where $T_{V_{J}}^{\mu \nu}$ contains the form factor set $f_{i}^{V_{J}}$. Using this generalization is particularly simple for inclusive observables obtained integrating over angular variables relative to the final states $\mathcal{F}$, such as the cross-section in eq. (2.6). In this case, the left-handed and righthanded currents do not interfere. It is also useful to distinguish between charged and neutral currents:

\footnotetext{
${ }^{2}$ In this paper we will use $i, j$ for flavour indices.
} 
Charged currents. Since the tree-level SM amplitude is purely left-handed, non-SM contributions due to right-handed charged currents do not interfere with the leading SM amplitude and can safely be neglected.

Neutral currents. In this case the result in eq. (2.6) can be generalized to

$$
\frac{\sigma^{\mathrm{BSM}}(\psi \bar{\psi} \rightarrow V h)}{\sigma^{\mathrm{SM}}(\psi \bar{\psi} \rightarrow V h)}=\frac{\left(g_{\psi}^{L}\right)^{2}}{\left(g_{\psi}^{L}\right)^{2}+\left(g_{\psi}^{R}\right)^{2}} R_{L}\left(q^{2}\right)+\frac{\left(g_{\psi}^{R}\right)^{2}}{\left(g_{\psi}^{L}\right)^{2}+\left(g_{\psi}^{R}\right)^{2}} R_{R}\left(q^{2}\right),
$$

with $R_{L}\left(R_{R}\right)$ identical to $R_{V}$ in eq. (2.6) but containing only the left-handed (righthanded) currents, and corresponding form factors.

\section{General EFT power counting for the form factors}

We can determine the power counting for the $q^{2}$ dependence of the different form factors in general. This is of interest, as the BSM momentum dependence present in the deformation of the amplitude from the SM case is the origin of the enhanced sensitivity to BSM effects in some kinematic distributions compared to the total integrated rate. This point was recently explored in some detail in refs. $[1,24]$ for the case of $h \rightarrow V \mathcal{F}$ decays.

For simplicity in this discussion, we focus on the case where the Higgs boson belongs to an $\mathrm{SU}(2)_{\mathrm{L}}$ doublet - in other words, to the case of a linear realization of the $\mathrm{SU}(2)_{\mathrm{L}} \times \mathrm{U}(1)_{\mathrm{Y}}$ gauge symmetry. The differences and similarities with respect to the non-linear realization of $\mathrm{SU}(2)_{\mathrm{L}} \times \mathrm{U}(1)_{\mathrm{Y}}$ will be discussed at the end of this section and in section 4 . We also limit ourself to evaluating the power counting associated with the dimensionality of the operators in the EFT expansion, ignoring the counting associated with powers of the gauge couplings. Our purpose here is to discuss some general properties of the form-factor expansion that just follow from the field content and Lorentz symmetry of the underlying theory.

\subsection{General decomposition}

As a first step, we expand each form factor around $q^{2}=m_{V}^{2}$ in the following way,

$$
f_{i}^{V_{J}}\left(q^{2}\right)=f_{i}^{V(0)}+\sum_{n \geq 1} f_{i}^{V_{J}(n)}\left(\frac{q^{2}-m_{V}^{2}}{m_{V}^{2}}\right)^{n}+\frac{q^{2}-m_{V}^{2}}{m_{V}^{2}} \Delta f_{i}^{V_{J}(\mathrm{LD})}\left(q^{2}\right),
$$

separating the constant terms, $f_{i}^{V(0)}$, the slopes, $f_{i}^{V_{J}(n \geq 1)}$, and the long distance contribution due to the propagation of real intermediate states, $\Delta f_{i}^{V_{J}(\mathrm{LD})}\left(q^{2}\right)$. We use the label $V_{J}$ $(V)$ to denote the form factors components that can (cannot) depend on the specific choice of the current.

By construction, the constant terms are associated with the $q^{2} \rightarrow m_{V}^{2}$ pole of the $h V \mathcal{F}$ amplitude. As such, they can be put in one-to-one correspondence with the general decomposition of a hypothetical on-shell $h V V$ amplitude (that is not kinematically accessible given the experimental values of $m_{h}$ and $m_{V}$ ), and select only the $J^{V}$ component of the current. The relation $f_{2}^{V}\left(m_{V}^{2}\right)=-f_{1}^{V}\left(m_{V}^{2}\right) / m_{V}^{2}$ implies $f_{2}^{V(0)}=-f_{1}^{V(0)} / m_{V}^{2}$ hence there are only three independent constant terms for each $V$ [1]. 
With a suitable choice of operator basis, the $f_{i}^{V_{J}(n \geq 1)}$ can always be associated with a $h V \mathcal{F}$ contact interaction. For this reason, the form factor slopes are dependent on the structure of the current creating the state $\mathcal{F}$. There are four independent $f_{i}^{V_{J}(n \geq 1)}$ for each $J$ and $n$.

Finally, $\Delta f_{i}^{V_{J}(\mathrm{LD})}\left(q^{2}\right)$ denote the sum of singular terms related to light poles in the amplitude. These poles are due to the electromagnetic current, for the $q^{2} \rightarrow 0$ pole, or the $q^{2} \rightarrow m_{\text {had }}^{2}$ poles of single hadronic states with mass $m_{\text {had }}$ and the same quantum number of $\mathcal{F}$. Note that there are other non-analytic terms due to multi-particle cuts.

\subsection{Constant terms}

To determine the power counting of the constant terms we start from the general decomposition of the $h V(\tilde{\epsilon}, p) V(\epsilon, q)$ amplitude:

$$
\mathcal{A}_{V V} \propto \tilde{\epsilon}_{\mu} \epsilon_{\nu}\left[f_{1}^{V(0)} g^{\mu \nu}+f_{3}^{V(0)}\left(p \cdot q g^{\mu \nu}-q^{\mu} p^{\nu}\right)+f_{4}^{V(0)} \epsilon^{\mu \nu \rho \sigma} p_{\rho} q_{\sigma}\right] .
$$

When considering the effective operators generating $\mathcal{A}_{V V}$ in a linear realization, they are constructed combining Higgs fields, $H \sim O(p)$, gauge stress tensors, $F^{\mu \nu} \sim O\left(p^{2}\right)$, and an arbitrary number of covariant derivatives, $D_{\mu} \sim O(p)$. $\mathrm{SU}(2)_{\mathrm{L}}$ invariance for this amplitude forbids terms with odd powers of $H$, and we need at least a $H H^{\dagger}$ pair to extract the physical Higgs-boson field $h$. Given this structure, $f_{1}^{V(0)}$ can be non-vanishing at $O\left(p^{4}\right)$ while $f_{3,4}^{V(0)}$ can be non-vanishing only at $O\left(p^{6}\right)$. Since within this EFT the only relevant $O\left(p^{4}\right)$ operator is the SM Higgs kinetic term, we obtain

$$
f_{1}^{V(0)}-f_{1}^{V(0) \mathrm{SM}}=O\left(p^{6}\right), \quad f_{3,4}^{V(0)}=O\left(p^{6}\right) .
$$

Here and in the following the notation $X=O\left(p^{n}\right)$ stands for: $X$ is non-vanishing at $O\left(p^{n}\right)$, irrespective of the dimension of $X$.

\subsection{Slopes in $q^{2}$ from contact interactions}

Contact interactions with a generic current $J_{\mu}$ generates an amplitude of the type

$$
\begin{aligned}
\mathcal{A}_{V}^{\mathcal{F}_{J}} \propto & \tilde{\epsilon}_{\mu} J_{\nu}^{\mathcal{F}_{J}}\left[f_{1}^{V_{J}(1)} g^{\mu \nu}+f_{2}^{V_{J}(1)} q^{\mu} q^{\nu}+f_{3}^{V_{J}(1)}\left(p \cdot q g^{\mu \nu}-q^{\mu} p^{\nu}\right)+f_{4}^{V_{J}(1)} \epsilon^{\mu \nu \rho \sigma} p_{\rho} q_{\sigma}\right] \\
& +O\left(q^{2}-m_{V}^{2}\right) .
\end{aligned}
$$

The currents in eq. (2.8) are $O\left(p^{3}\right)$ and $\mathrm{SU}(2)_{\mathrm{L}}$ invariant. The $\mathrm{SU}(2)_{\mathrm{L}}$ invariance of the amplitude then implies at least two Higgs fields, and the counting of derivatives and/or gauge fields, both of $O(p)$, implies that $f_{1}^{V_{J}(1)}$ can be generated at $O\left(p^{6}\right)$, while $f_{2,3,4}^{V_{J}(1)}$ can appear only at $O\left(p^{8}\right)$. The generalization to higher slopes can easily be obtained noting that the extra $q^{2}$ dependence implies (at least) two more derivatives:

$$
f_{1}^{V_{J}(n \geq 1)}=O\left(p^{4+2 n}\right), \quad f_{2,3,4}^{V_{J}(n \geq 1)}=O\left(p^{6+2 n}\right) .
$$

The decomposition (2.8) allow us to consider also scalar currents of the type $\bar{\psi}_{L(R)} \psi_{R(L)}$, with a covariant derivative acting on one (or both) fermion fields. In this 
case the power counting is the same as above: $\mathrm{SU}(2)_{\mathrm{L}}$ invariance implies an odd power of Higgs fields, but the structure of the current is such that $J_{\nu}^{\mathcal{F}_{J}}$ is at least of $O\left(p^{4}\right)$ with a covariant derivative acting on the fermion fields. By construction, our decomposition of the amplitude does not allow us to include local operators with a generic tensor current $\left(\bar{\psi}_{L(R)} \sigma^{\mu \nu} \psi_{R(L)}\right) .^{3}$

\subsection{The photon pole}

In most realistic BSM constructions, the only relevant additional pole in the amplitude is the one occurring at $q^{2}=0$ due the photon propagator, for states $\mathcal{F}$ such that $\left\langle\mathcal{F}\left|J_{\mu}^{\mathrm{em}}\right| 0\right\rangle \neq 0$, where $J_{\mu}^{\mathrm{em}}$ is the electromagnetic current. In this limit, the structure of $\Delta f_{i}^{V_{J}(\mathrm{LD})}\left(q^{2}\right)$ can be put in one-to-one correspondence to the general decomposition of the on-shell $h Z_{\gamma}$ amplitude $\left(q^{2}\right.$ dependent terms that cancel the pole at $q^{2}=0$ can be absorbed in the form factor slopes). The latter can be decomposed in terms of two independent Lorentz structures,

$$
\mathcal{A}_{Z \gamma} \propto \tilde{\epsilon}_{\mu} \epsilon_{\nu}\left[g_{3}\left(p \cdot q g^{\mu \nu}-q^{\mu} p^{\nu}\right)+g_{4} \epsilon^{\mu \nu \rho \sigma} p_{\rho} q_{\sigma}\right] .
$$

Within the SM the effective couplings $g_{3,4}$ are different from zero only beyond tree level. This fact does not imply that such effects must be loop suppressed in a BSM sector. Within the EFT approach, using the same power counting adopted for the $\mathcal{A}_{V V}$ amplitude, it follows that $g_{3,4}$ can be different from zero starting at $O\left(p^{6}\right)$. The resulting $\Delta f_{i}^{Z_{J}(\mathrm{LD})}\left(q^{2}\right)$ does depend on the nature of $\mathcal{F}$; however, the dependence is fully specified by the electric charge $Q_{\psi}$ of the fermions in $|\mathcal{F}\rangle=|\bar{\psi} \psi\rangle$ :

$$
\Delta f_{3,4}^{Z_{J}(\mathrm{LD})}\left(q^{2}\right) \propto g_{3,4} \frac{Q_{\psi}}{q^{2}}=O\left(p^{6}\right), \quad \Delta f_{1,2}^{Z_{J}(\mathrm{LD})}\left(q^{2}\right)=0
$$

\subsection{Summary}

Due to the above discussion, the power counting within the linear EFT approach implies the following general expansion of the form factors up to $O\left(p^{6}\right)$ :

$$
\begin{aligned}
f_{1}^{V_{J}(0)}-f_{1}^{V_{J}(0) \mathrm{SM}} & =a_{V}^{(1)} \frac{v^{2}}{\Lambda^{2}}+b_{V_{J}}^{(1)} \frac{q^{2}-m_{V}^{2}}{\Lambda^{2}}, \\
f_{i}^{V(0)}-f_{i}^{V(0) \mathrm{SM}} & =a_{V}^{(i)} \frac{1}{\Lambda^{2}}, \quad(i=2,3,4), \\
\Delta f_{3,4}^{Z_{J}(\mathrm{LD})}-\Delta f_{3,4}^{Z_{J}(\mathrm{LD}) \mathrm{SM}} & =c_{Z}^{(i)} Q_{\psi} \frac{v^{2}}{\Lambda^{2}} \frac{1}{q^{2}}, \quad(i=3,4),
\end{aligned}
$$

where the $a_{V}^{(1)}, b_{V_{J}}^{(1)}$, and $c_{Z}^{(i)}$ are $O(1)$ couplings.

As we will explicitly illustrate in the next section, a very similar expansion is recovered also in the non-linear case. This is somewhat expected. Both theories are defined by derivative expansions with the same field content and the appearance of the momentum dependence is constrained by Lorentz invariance. As such, the only form factor that can

\footnotetext{
${ }^{3}$ In general, contributions due to tensor currents do not need not be loop suppressed [25]. However, their inclusion require an extension of the present formalism that is beyond the scope of the present work.
} 
have $q^{2}$ dependence at sub-leading order is $f_{1}^{V(0)}$. In this manner, although the linear realization was used in this discussion, the structure of the form factors that has been identified is more general in many respects. This is a reason why a general analysis in terms of a form factor decomposition is a useful intermediate step until the most suitable underlying EFT is experimentally clarified.

The form factor formalism can also accommodate a non-SM momentum dependence (consistent with crossing symmetry and Lorentz invariance) that differs from the polynomial expansion around $m_{V}^{2}$ of the $f_{i}$ that we have assumed. The assumed dependence is suitable for a local EFT expansion of the form factors. Non-SM momentum dependence, with unsuppressed coefficients will generally lead to unitarity violation that is proximate to the scale $\sim v$. We restrict our attention to a polynomial derivative expansion of the form factors in what follows, as appropriate for local EFTs with no additional light states. We also generally consider suppressed coefficients in the derivative expansion of the momentum dependence of the form factors for this reason. See the discussion in section 5.2 for more details on this point.

As far as the decomposition of the form factors is concerned, a key difference between the two EFT approaches concerned the leading term, $f_{1}^{V(0)}=-m_{V}^{2} f_{2}^{V(0)}$, i.e. the only term that does not vanish at the tree level within the SM. Within the linear EFT approach, the only leading operator contributing to the $h V \mathcal{F}$ amplitude at the tree level is the SM Higgs kinetic term. This fact is responsible for the absence of $O(1)$ corrections in $f_{1}^{V(0)}$. Within the non-linear case, the SM is recovered only with an explicit tuning of the leading operators. In the absence of such tuning, $f_{1}^{V(0)}$ can receive $O(1)$ corrections in the nonlinear EFT approach compared to the SM expectation.

\section{The form factors in explicit EFTs}

We now decompose the form factors in eq. (3.1) in terms of local operators in an Effective Lagrangian. This is a necessary step to calculate the amplitude beyond tree level in a BSM scenario. However, as we have emphasized, there is more than one EFT to choose from. In the following, we provide an illustrative decomposition of the form factors in two EFTs of particular current interest: an EFT with a non-linear realization of $\mathrm{SU}(2)_{\mathrm{L}} \times \mathrm{U}(1)_{\mathrm{Y}}$, where the massive Higgs-like boson $h$ is introduced as an $\mathrm{SU}(2)_{\mathrm{L}} \times \mathrm{U}(1)_{\mathrm{Y}}$ scalar singlet $[26,27]$ (see also ref. [28]), and the linear realization EFT where $h$ belongs to an $\mathrm{SU}(2)_{\mathrm{L}}$ doublet $H$. As already emphasized, these two EFT approachs are in fact distinct. We comment about the differences that arise in the specific case of the $h V \mathcal{F}$ form factors at the end of this section.

In the following discussion, our aim is not to provide a systematic evaluation of the form factors in these two theories including all terms in the sub-leading operator basis. In both cases, the number of sub-leading operators is sizable, and such an analysis is not particularly illuminating. Instead, we restrict our attention to contributions to the physical process due to anomalous Higgs-like Boson interactions that are illustrative of non-SM $q^{2}$ dependence in the form factors. In particular, we neglect local interactions with currents different from $J^{V}$, and possible contributions due to a modified $h Z_{\gamma}$ amplitude. 


\subsection{Non-Linear realization of $\mathrm{SU}(2)_{\mathrm{L}} \times \mathrm{U}(1)_{\mathrm{Y}}$}

The non-linear EFT is based on the assumption of a global symmetry $\mathcal{G}=\mathrm{SU}(2)_{\mathrm{L}} \times$ $\mathrm{SU}(2)_{\mathrm{R}}$ existing in a BSM sector, that is spontaneously broken into the diagonal subgroup $\mathrm{SU}(2)_{\mathrm{L}+\mathrm{R}}$ (corresponding to custodial symmetry). The Goldstone bosons resulting from this spontaneous symmetry breaking, denoted by $\pi^{a}$, are described by the field

$$
\Sigma(x)=e^{i \sigma_{a} \pi^{a} / v} \quad a=1,2,3,
$$

that transforms (linearly) as a $(2, \overline{2})$ of $\mathcal{G}$. The $\mathrm{SU}(2)_{\mathrm{L}} \times \mathrm{U}(1)_{\mathrm{Y}}$ subgroup of $\mathrm{SU}(2)_{\mathrm{L}} \times \mathrm{SU}(2)_{\mathrm{R}}$ is weakly gauged, such that the $\pi^{a}$ can be identified with the longitudinal components of the SM massive gauge bosons. The SM gauge symmetry is nonlinearly realized. An $h$ field is introduced as a massive $0^{+}$scalar, which is a singlet under $\mathcal{G}$. The leading order terms involving the observed scalar field in the derivative expansion of this EFT are

$$
\begin{aligned}
\mathcal{L}_{E W h}^{(2)} & =\mathcal{L}_{h}+\mathcal{O}_{L O}+\mathcal{O}_{Y}, \\
\mathcal{L}_{h} & =\frac{1}{2}\left(\partial_{\mu} h\right)^{2}-\frac{1}{2} m_{h}^{2} h^{2}\left[1+d_{3}\left(\frac{h}{v}\right)+\frac{d_{4}}{4}\left(\frac{h}{v}\right)^{2}+\cdots\right] \\
\mathcal{O}_{L O} & =\frac{v^{2}}{4}\left[1+2 c_{1}\left(\frac{h}{v}\right)+\cdots\right] \operatorname{Tr}\left[\left(D_{\mu} \Sigma\right)^{\dagger} D^{\mu} \Sigma\right] .
\end{aligned}
$$

Here $\mathcal{O}_{Y}$ are the Yukawa interactions. The coefficients have been normalized to define the suppression scale in the expansion in powers of $h$ as $1 / v$. The most famous UV completion of this theory is the singular case where the coefficients in the $(h / v)$ expansion are fixed such that $h$ and the Goldstone bosons can be combined into a single linear multiplet $H=(1+h / v) \Sigma$. In this case $\mathcal{L}_{E W h}^{(2)}$ coincides with the SM Higgs boson Lagrangian. For the purpose of this work, the unknown coefficients present in $\mathcal{L}_{E W h}^{(2)}$ are arbitrary numerical parameters subject to experimental constraints: present data suggests their values are proximate to the SM limit, but we are far from having ruled out a more general framework. The presence of a light scalar in the spectrum can postpone the usual unitarity problems of massive vector bosons to significantly higher scales. Considering LHC's discovery reach, a true "Higgs-like Boson", proximate in its properties to the SM Higgs boson, and described by this theory, is a consistent EFT framework.

The complete list of local operators to the next order in the derivative expansion, under the hypothesis of Minimal Flavour Violation [29-32], can be found in ref. [33, 34]. Here we restrict the attention to operators that are CP even and do not contain any source of custodial symmetry breaking. ${ }^{4}$ In this limit, only two $O\left(p^{4}\right)$ operators lead to a non-vanishing $h V V^{*}$ amplitude at the tree level:

$$
\begin{aligned}
\mathcal{L}_{E W h}^{(4)} & =\sum_{i=W, W \partial h, \ldots} c_{i}^{(4)} \mathcal{O}_{i} \\
\mathcal{O}_{W} & =g_{2} D_{\mu} W_{a}^{\mu \nu} \operatorname{Tr}\left(\Sigma^{\dagger} i \sigma^{a} \overleftrightarrow{D}_{\nu} \Sigma\right) F_{W}, \\
\mathcal{O}_{W \partial h} & =g_{2} W_{a}^{\mu \nu} \operatorname{Tr}\left(\Sigma^{\dagger} i \sigma^{a} \overleftrightarrow{D}_{\mu} \Sigma\right) \partial_{\nu} F_{W \partial H}
\end{aligned}
$$

\footnotetext{
${ }^{4}$ More precisely, we neglect operators that either have a $\sigma_{3}$ in a trace, are proportional to the hypercharge coupling $\left(g_{1}\right)$, or are related to SM fermion masses through the equations of motion.
} 
Here the $F_{i}$ are generic polynomials in $(h / v)^{n}$ and we have used the notation

$$
\begin{aligned}
D_{\mu} \Sigma & =\partial_{\mu} \Sigma+\frac{i g_{2}}{2} W_{\mu}^{a} \sigma_{a} \Sigma-\frac{i g_{1}}{2} B_{\mu} \Sigma \sigma_{3} \\
\Sigma^{\dagger} \sigma^{a} \overleftrightarrow{D}_{\nu} \Sigma & =\Sigma^{\dagger} \sigma^{a}\left(D_{\nu} \Sigma\right)-\left(D_{\nu} \Sigma\right)^{\dagger} \sigma^{a} \Sigma .
\end{aligned}
$$

Considering also the leading order operator $\left(\mathcal{O}_{L O}\right)$, we have three operators generating a non-vanishing $h V V^{*}$ amplitude at the tree level. After expanding the $(h / v)^{n}$ polynomials to the first order, to be consistent with our past work [1] we define the Wilson coefficients $c_{i}$ for the $h$-linearized terms,

$$
\begin{aligned}
\hat{\mathcal{O}}_{L O} & =\frac{v c_{1}}{2} h \operatorname{Tr}\left[\left(D_{\mu} \Sigma\right)^{\dagger} D^{\mu} \Sigma\right] \\
\hat{\mathcal{O}}_{W} & =\frac{g_{2} c_{2}}{v} h D_{\mu} W_{a}^{\mu \nu} \operatorname{Tr}\left[\Sigma^{\dagger} i \tau^{a} \overleftrightarrow{D}_{\nu} \Sigma\right] \\
\hat{O}_{W \partial H} & =\frac{g_{2} c_{3}}{v}\left(\partial_{\nu} h\right) W_{a}^{\mu \nu} \operatorname{Tr}\left[\Sigma^{\dagger} i \tau^{a} \overleftrightarrow{D}_{\mu} \Sigma\right]
\end{aligned}
$$

such that the SM case correspond to $c_{i}^{\mathrm{SM}}=(1,0,0)$. The form factor basis expanded in terms of these operators is

$$
\begin{array}{ll}
f_{1}^{V}\left(q^{2}\right)=c_{1}+g_{2}^{2}\left(c_{2}+c_{3}\right)\left(1+\frac{q^{2}}{m_{V}^{2}}\right), & f_{2}^{V}\left(q^{2}\right)=-\frac{1}{m_{V}^{2}}\left[c_{1}+2 g_{2}^{2}\left(c_{2}+c_{3}\right)\right], \\
f_{3}^{V}\left(q^{2}\right)=\frac{2 g_{2}^{2}}{m_{V}^{2}} c_{3}, & f_{4}^{V}\left(q^{2}\right)=0 .
\end{array}
$$

As can be seen, this result is consistent with the general decomposition in eq. (3.10), provided we assume $c_{2,3}$ and $\left(c_{1}-1\right)$ to be $O\left(v^{2} / \Lambda^{2}\right)$, as expected by naive dimensional analysis. A detailed analysis of the naive power counting in this EFT has recently been presented in refs. [34, 35], where it has been shown that $\left(c_{1}-1\right)$ could be of $O(1)$ without spoiling the consistency of the loop expansion.

Beside naive dimensional analysis, the expectation for the size of these parameters must balance several dynamical considerations. First of all, we stress that in a nonlinear chiral EFT the cut off scale can be lower than its naive value, $\Lambda \sim 4 \pi v$ [36]. In the presence of strong interactions with a light $0^{+}$state, large flavour, colour or Goldstone boson groups of dimension $\sim N$ in the underlying theory can lead to a cut off scale of $\Lambda \sim 4 \pi v / \sqrt{N}[37-39]$.

One can form more specific expectations for the unknown parameters $\left(c_{1}, c_{2}, c_{3}\right)$ at the cost of introducing model dependence and moving outside of a totally general EFT framework. For example, in the case of a composite Higgs (and the particular benchmark models discussed in ref. [20]) one expects $\left(c_{1}-1\right)=O\left(v^{2} / f^{2}\right)$, while $c_{2,3}=O\left(v^{2} / M^{2}\right)$. Here $M$ is the mass of the spin-1 resonances in the strongly interacting sector, that is related to the scale $f$ by $M \sim g_{\star} f$ with a coupling $g_{\star}$ expected to be in the range $1 \leq g_{\star} \leq 4 \pi$. The experimental constraints on $f$ and $M$ are model dependent, but have strengthened significantly after run-I at LHC. Estimates of the bounds on these parameters [20] imply $v^{2} / f^{2} \lesssim 0.1$ in these benchmark scenarios. The size of the deviations shown in figure 3 can be compared to this benchmark expectation. 
Adopting a bottom-up EFT approach allows us not to be overly concerned with these model considerations for the sake of a phenomenological analysis. In section 5 we will analyse in detail the range $O\left(10^{-2}-10^{-1}\right)$, that we consider a rather natural choice for these parameters (the parameters are chosen in the range of 0.01 to 0.2 for most of the figures shown in figure 3). We also make a few illustrative plots for $O(0.1-1)$ values of the coefficients, in particular in the case labeled suppressed SM couplings in figure 3, which we argue is likely to be already excluded by the total signal strength measurements of ATLAS and CMS.

\subsection{Linear realization of $\mathrm{SU}(2)_{\mathrm{L}} \times \mathrm{U}(1)_{\mathrm{Y}}$}

In the case of the linear EFT, the lowest-order Lagrangian coincides with the SM Lagrangian and the Higgs mechanism is at work to generate the masses of the observed (non scalar) SM particles. The complete (yet minimal) set of operators appearing at dimensionsix (i.e. the first non-trivial order in the EFT expansion for higher dimensional operators contributing to processes conserving total lepton number) has been presented in ref. [40]. Following the notation of ref. [40], the two operators modifying the $h V V^{*}$ amplitude at tree level, in the limit of unbroken $\mathrm{CP}$ and custodial symmetries, are

$$
\mathcal{P}_{W W}=\frac{g_{2}^{2}}{\Lambda^{2}} H^{\dagger} H W_{\mu \nu}^{a} W^{a \mu \nu}, \quad \mathcal{P}_{\square}=\frac{2}{\Lambda^{2}}\left(H^{\dagger} H\right) \square\left(H^{\dagger} H\right),
$$

and these operators lead to the decomposition

$$
\begin{array}{rlrl}
f_{1}^{V}\left(q^{2}\right) & =1+\frac{v^{2} c_{\square}}{\Lambda^{2}}, & & f_{2}^{V}\left(q^{2}\right)=-\frac{1}{m_{V}^{2}}\left(1+\frac{v^{2} c_{\square}}{\Lambda^{2}}\right), \\
f_{3}^{V}\left(q^{2}\right)=\frac{g_{2}^{2}}{m_{V}^{2}}\left(\frac{v^{2} c_{W W}^{V}}{\Lambda^{2}}\right), & f_{4}^{V}\left(q^{2}\right)=0 .
\end{array}
$$

By construction, the $c_{i}$ are dimension-less couplings expected to be $O(1)$. Given the definition of the operator $\mathcal{P}_{W W}$, an explicit $V$ dependence does appear in its contribution to $f_{3}^{V}: c_{W W}^{V}=\left\{c_{W W}, \cos ^{2} \theta_{W} c_{W W}\right\}$. However, unbroken custodial symmetry is consistently recovered in the limit $g_{1} \rightarrow 0$.

In the operator basis of ref. [40], the momentum dependence in the form factors is generated by the contact operators,

$$
\begin{aligned}
\mathcal{P}_{H J} & =\frac{4}{\Lambda^{2}}\left(H^{\dagger} i \overleftrightarrow{D}_{\mu}^{I} H\right) J^{\mu}, \\
J^{\mu} & =\left\{\bar{Q}_{L} \sigma_{I} \gamma^{\mu} Q_{L}, \quad \bar{Q}_{L} \sigma_{3} \gamma^{\mu} Q_{L}, \quad \bar{u}_{R} \gamma^{\mu} u_{R}, \quad \bar{d}_{R} \gamma^{\mu} d_{R},\right. \\
\bar{L}_{L} \sigma_{I} \gamma^{\mu} L_{L}, & \left.\bar{L}_{L} \sigma_{3} \gamma^{\mu} L_{L}, \quad \bar{e}_{R} \gamma^{\mu} e_{R}\right\}
\end{aligned}
$$

With a proper choice of couplings, such that the effective charged and neutral current combinations coincides with $J_{V}^{\mu}$ in eq. (2.1), one generates a momentum dependence in $f_{3}^{V}$, with correlated constant shift in $f_{2}^{V}$, as in eq. (4.10). 


\subsection{Linear vs. Nonlinear realization of $\mathrm{SU}(2)_{\mathrm{L}} \times \mathrm{U}(1)_{\mathrm{Y}}$}

Despite the similarities of the decomposition of the form factors into the linear and nonlinear EFT frameworks, it is important to realize that, at any fixed order in the EFT expansion, these theories are in fact distinct EFTs. ${ }^{5}$ This is perhaps counterintuitive, given the two theories have exactly the same gauge symmetry and the same field content. Which might naively be assumed to be sufficient to establish the equivalence of the two theories. One should also note that a comparison of the number of free parameters (at a fixed order in the EFT expansion) contributing to a subset of processes, while ignoring correlated constraints due to the different (global) symmetry structure in the two theories, is also insufficient to establish the equivalence of the two approaches. One way to see this is as follows.

Consider the analysis of $h \rightarrow V \ell^{+} \ell^{-}$decays of refs. [1,24], where it was emphasized that studies of the $d \Gamma / d q^{2}$ spectrum are a sensitive probe of BSM effects compared to the total rate. As discussed in section 4.2, within the linear EFT the non-standard $q^{2}$ dependence in the form factors is generated from contact operators of the type $\mathcal{P}_{H J}$. These operators contribute also to the (on-shell) $Z \rightarrow \ell^{+} \ell^{-}$decay when both Higgs doublets get a vev. As such, their Wilson coefficients are constrained by the electroweak precision tests performed at LEP. This implies that in the linear EFT, the effects of anomalous Higgs couplings present in the $d \Gamma / d q^{2}$ spectrum is correspondingly constrained by LEP measurements [41].

Now consider the nonlinear EFT. In this case the BSM momentum dependence in $h \rightarrow V \ell^{+} \ell^{-}$is not directly related to deviations in $Z \rightarrow \ell^{+} \ell^{-}$as the scalar is not embedded in an $\mathrm{SU}(2)_{\mathrm{L}}$ doublet. In other words, in this EFT the contact operators with different powers of the $h$ field are unrelated in general. As a result, studies of the $d \Gamma / d q^{2}$ spectrum are in fact (fairly unique) probes of Green's function with one external $h$ field, and this spectrum is not as directly constrained by existing electroweak precision measurements of $Z \rightarrow \ell^{+} \ell^{-}$decay. Considering correlated constraints, the range of allowed parameters in the linear realization is a subset of the parameters that are still experimentally allowed in the non linear EFT.

\section{Associated production as a probe of anomalous couplings}

In this section we consider the constraints that current and future associated production data can place on the parameters in the EFTs. As a starting hypothesis, we assume that by means of appropriate cuts (and background subtraction), the LHC experiments will be able to isolate and measure the $\sigma(q \bar{q} \rightarrow V h)$ s-channel cross section. Our main aim is to illustrate the utility of reporting the measured associated production spectrum as a function of the reconstructed $V h q^{2}$ distribution for constraining the underlying form factors, and hence the EFTs.

\subsection{Current data}

Current associated production data reported by ATLAS [42] and CMS [43] can be summarized as follows. ATLAS reports a 95\% C.L. limit on the signal strength characterizing

\footnotetext{
${ }^{5} \mathrm{~A}$ general discussion about the similarities and differences of these two EFT will be presented elsewhere.
} 
this process of $\{1.8,3.4\}$, normalized to the SM, for operating energies of $\{7,8\} \mathrm{TeV}$ and assuming $m_{h}=125 \mathrm{GeV}$. This compares to an expected limit of $\{3.3,2.5\}$ for these operating energies. Expressed in terms of extracted signal strengths $(\hat{\mu})$ the reported values are $\{-2.7 \pm 1.6,1 \pm 1.4,-0.4 \pm 1.1\}$ for $\{7,8,7+8\} \mathrm{TeV}$ operating energies. Here we have added the reported error in quadrature. Similarly, CMS reports an observed 95\% C.L. limit of 1.89 compared to an expected limit of 0.95 for $m_{h}=125 \mathrm{GeV}$ using the combined $7+8 \mathrm{TeV}$ data set. The signal strength corresponding to the $2.1 \sigma$ local excess is reported to be $\hat{\mu}=1.0 \pm 0.5$ for $m_{h}=125 \mathrm{GeV}$.

At present is not easy to translate these results into precise constraints on the form factors (or the EFT parameters). In reporting a value for the signal strength in associated production, the kinematics of the various events that are defined to correspond to the signal strength are averaged over. In particular, what is reported effectively averages over a value of $q^{2}$,

$$
\hat{\mu} \equiv \int d q^{2} R_{V}\left(q^{2}\right) \times \mathcal{B}_{b}
$$

for the events that pass the selection cuts. Here $\mathcal{B}_{b}=B R_{\mathrm{BSM}}(h \rightarrow \bar{b} b) / B R_{\mathrm{SM}}(h \rightarrow \bar{b} b)$. As can be seen by the from of eq. (2.6), the averaging over $q^{2}$ is particularly relevant for the sensitivity to different combinations of the underlying parameters in the EFT. There is clearly more information in reporting $d \sigma / d q^{2}$ than in the averaged total signal strength.

To see the sensitivity that the spectrum offers on the underlying parameters, we normalize the average $\bar{q}^{2}$ of the $V h$ final state as $\bar{q}^{2}=\mathcal{N} m_{h}^{2}(1+\sqrt{\rho})^{2}$. Here $\rho=m_{V}^{2} / m_{h}^{2}$. There is an effective value of $\bar{q}^{2}$ that corresponds to a reported value of $\hat{\mu}$ for the total signal strength in associated production. Such a parameter is not reported by ATLAS and CMS, although clearly $\mathcal{N} \sim \mathcal{O}(1)$ and $\mathcal{N} \geq 1$. We strongly encourage the experimental collaborations to report a value for $\bar{q}^{2}$ when any total associated production signal strength is reported in the future, and to bin the data in terms of different effective reconstructed $\bar{q}^{2}$ once their are sufficient events.

It is easy to illustrate the value of such a binning. Consider the case where the signal events are dominantly at threshold, so that $\mathcal{N} \approx 1$. This is the naive expectation, given the cross section falls rapidly due to the PDF behavior. In this case, employing the form factor parameterization in eq. (4.10) and expanding to the cross section to leading order in $g_{2}^{2}$, we would obtain the following constraint

$$
c_{1}^{2}+2 c_{1} g_{2}^{2}\left(c_{2}+c_{3}\right)\left(1+\frac{(1+\sqrt{\rho})^{2}}{\rho}\right)+4 c_{1} c_{3}^{\star} g_{2}^{2}\left(1+\frac{1}{\sqrt{\rho}}\right)=\hat{\mu} \mathcal{B}_{b}^{-1} .
$$

Conversely, considering a large $\mathcal{N}$ bin in associated production, would lead to

$$
2 \mathcal{N} c_{1} g_{2}^{2}\left(c_{2}+c_{3}\right) \frac{(1+\sqrt{\rho})^{2}}{\rho}=\hat{\mu} \mathcal{B}_{b}^{-1}
$$

at leading order in $1 / \mathcal{N}$. As can be seen, a different set of Wilson coefficients is constrained in the cases $\mathcal{N} \sim 1$ and $\mathcal{N} \gg 1$. Given the lack of information about the $\bar{q}^{2}$ corresponding to present data, and the fact the reported $\hat{\mu}$ values are still affected by $O(1)$ errors, in the following we will neglect the present experimental constraints. 

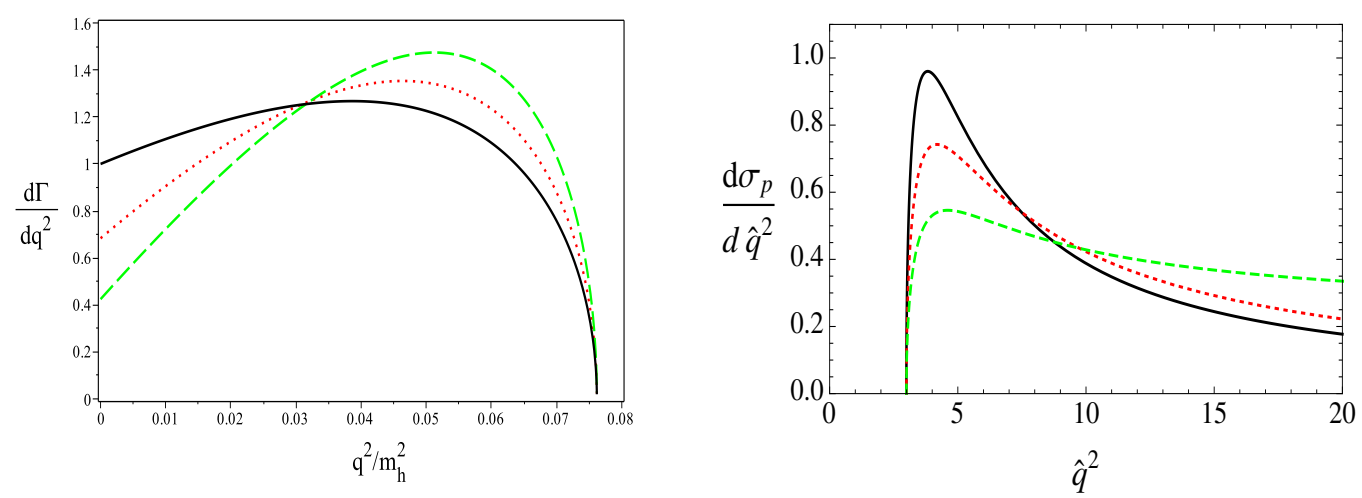

Figure 1. Left: $\mathrm{d} \Gamma\left(h \rightarrow Z \ell^{+} \ell^{-}\right) / \mathrm{d} \hat{q}^{2}$ spectra $\left(\hat{q}^{2}=m_{\ell \ell}^{2} / m_{h}^{2}\right)$, in arbitrary units, for different values of the EFT parameters chosen to leave the total $h \rightarrow Z \ell^{+} \ell^{-}$rate unchanged. The values were not chosen so that the total integrated associated production cross section is the same. The black (full) curve corresponds to the SM, $c_{i}=(1,0,0)$, the red (dotted) curve is for $c_{i}=(0.82,-0.8,0.8)$, the green (dashed) curve for $c_{i}=(0.06,0,1.4)$. Right: the partonic $d \sigma(q \bar{q} \rightarrow Z h) / d \hat{q}^{2}$ for the same EFT parameter choices (again arbitrary units). For the sake of presentation, in the right curve the \{black, red, green $\}$ curves have been multiplied by a factor $\{5,1,2.7\}$.

\subsection{Future associated production spectra}

Eventually, with sufficient data, a binned associated production spectra as a function of $q^{2}$ can be experimentally constructed. In this section, we report some simple numerical examples of the importance of such spectra in constraining the underlying EFT.

The kinematic distributions of associated production offer a complementary sensitivity to the unknown physics parameters. This is expected as the various distributions probe different off-shell momentum regions, as previously noted. In figure 1 (right) we show the effect of varying the form factors in the $Z h$ associated partonic production cross section at fixed $\hat{q}^{2}=q^{2} / m_{h}^{2}$. We use the form factor parameterization in eq. (4.10) and we restrict our detailed analysis to the case of the $Z h$ production, given the $q^{2}$ dependence of $\sigma\left(\bar{\psi}_{i} \psi_{j} \rightarrow W h\right)$ and $\sigma\left(\bar{\psi}_{i} \psi_{i} \rightarrow Z h\right)$ are qualitatively very similar. In $Z h$ production, the start of the distribution, corresponding at $\vec{p}_{h}=0$, occurs at the partonic value $\hat{q}^{2}=$ $\left(1+\sqrt{m_{Z}^{2} / m_{h}^{2}}\right)^{2} \approx 3 .{ }^{6}$ For comparison, in figure 1 (left) the $\mathrm{d} \Gamma\left(h \rightarrow Z \ell^{+} \ell^{-}\right) / \mathrm{d} q^{2}$ spectra $\left(q^{2}=m_{\ell \ell}^{2}\right)$ for the same set of $c_{i}$ is also shown.

The three sets of $c_{i}$ used in figure 1 have been chosen such that they give rise to the same $\Gamma\left(h \rightarrow V \ell^{+} \ell^{-}\right)$rate. In associated production the introduction of a non-SM $q^{2}$ dependence has a significantly larger impact, and it affects both the total cross section and the $d \sigma / d \hat{q}^{2}$ spectrum. To better illustrate the difference in the $q^{2}$ distribution, in figure 1 we have adopted a different overall rescaling of the three associated production distributions, multiplying the $\{$ black, red,green $\}$ curves by $\{5,1,2.7\}$. The corresponding total cross sections obtained after convoluting with $\mathrm{PDF}^{\prime}{ }^{7}$ are $\sim\{0.4,2.3,0.9\}[\mathrm{pb}]$ for

\footnotetext{
${ }^{6}$ We use the numerical values $m_{h}=125 \mathrm{GeV}, m_{Z}=91.2 \mathrm{GeV}, \alpha_{\mathrm{em}}\left(m_{Z}\right)=1 / 128.93$ and $\sin ^{2} \theta_{W}=0.231$.

${ }^{7}$ Normalizing our leading order result to the value of the total cross section as given by the Higgs xsection working group.
} 
$m_{h}=125 \mathrm{GeV}$. The presence of a non-SM $q^{2}$ dependence is clearly evident already from the values of total cross sections, but the differential distribution provides independent information to decipher the underlying dynamics.

The extra sensitivity of the associated production spectra to possible non-SM $q^{2}$ dependence of the form factors comes at a cost in terms of predictivity of an EFT derivative expansion, if $c_{2,3},\left|c_{1}-1\right|$ are of order one. The general expression in eq. (2.6) in terms of form factors makes no assumptions about the underlying EFT, and is valid for arbitrary values of $q^{2}$. However, the EFT expansion of the form factors in eq. (4.10) is based on a derivative expansion that breaks down for $q^{2} \sim v^{2} /\left|c_{i}-c_{i}^{\mathrm{SM}}\right|$, i.e. almost immediately above threshold if $c_{2,3},\left|c_{1}-1\right| \sim O(1)$. This failure of the EFT expansion is signaled by the increasing difference (at large $q^{2}$ ) between the general decomposition in eq. (2.6) -supplemented by the polynomial form factors in eq. (4.10)- and its truncated expression,

$$
\frac{\sigma^{\mathrm{EFT}}(\bar{\psi} \psi \rightarrow Z h)}{\sigma^{\mathrm{SM}}(\bar{\psi} \psi \rightarrow Z h)}=c_{1}^{2}+2 g_{2}^{2} c_{1}\left(c_{2}+c_{3}\right)\left(1+\frac{q^{2}}{m_{Z}^{2}}\right)+6 g_{2}^{2} c_{1} c_{3}\left(\frac{q^{2}+m_{Z}^{2}-m_{h}^{2}}{\left|\overrightarrow{p_{h}}\right|^{2}+3 m_{Z}^{2}}\right)
$$

where we have neglected all $q^{4} / m_{V}^{4}$ terms. For the illustrative $c_{i}$ adopted in figure 1 this difference exceed $50 \%$ already for $q^{2}>5 m_{h}^{2}$ given we have chosen values of the $c_{i}$ with $O(1)$ deviations from the SM. The large difference between truncated and non-truncated expressions for these sets of $c_{i}$ is shown by the comparison of upper-left and middle-left panels in figure 3: we stress that none of the two panels can be considered as a realistic benchmark scenario, given higher-order terms are necessarily relevant. This reinforces the need to perform the analysis in terms of general form factors in case of sizable deviations from the SM already at low $q^{2}$ values. Conversely, the derivative expansion in terms of local operators is predictive and under control for the more "natural" values of the Wilson coefficients adopted in the middle and right panels in figure 3 , as explicitly illustrated by the plots in the middle row of figure 3 .

It is also important to note that the partonic invariant mass distribution is not directly accessible in experiments at hadron colliders. Two key ingredients are necessary in order to provide a distribution closer to what can be measured at the LHC: i) the convolution of the partonic cross section with the parton distribution functions (PDF's); ii) an estimate of how the error on the reconstructed final state four momenta limits the experimental sensitivity to the Wilson coefficients. We discuss each of these effects in turn in the following sections.

\subsection{Breit-Wigner smearing and experimental reconstruction uncertainty}

The experimental uncertainty on the reconstructed $p$ and $p_{h}$ four vectors have to be taken into account when accessing the experimental sensitivity of various distributions. We provide a first rough estimate of this uncertainty by convoluting the theoretical distribution with Breit-Wigner momentum distributions for $p$ and $p_{h}$, treating the corresponding $\Gamma_{h, V}$ not as a fundamental widths but as an experimental error associated with the momentum reconstruction. More explicitly, we first express the theoretical distribution as a function of $p^{2}=m_{V}^{2}$ and $p_{h}^{2}=m_{h}^{2}$, we then convolute this distribution with appropriate Breit-Wigner for these two kinematical variables. In both cases we assume an effective experimental error leading to $\Gamma_{h, V} \sim O(10 \mathrm{GeV})$. 

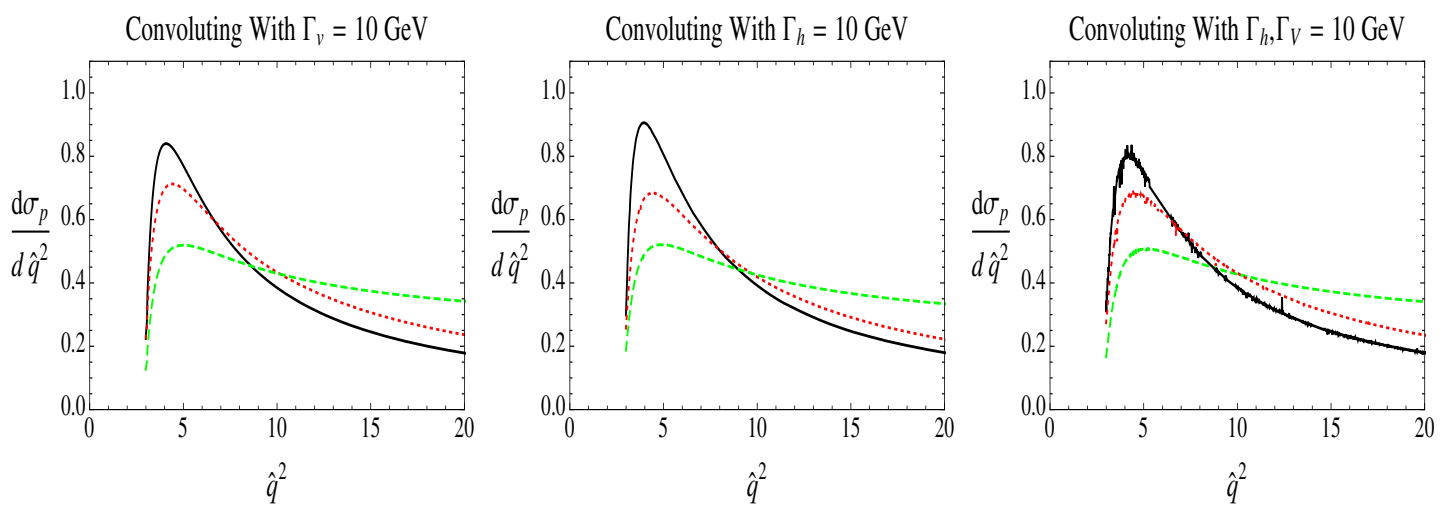

Figure 2. Left: convolution of the partonic $q^{2}$ spectrum with a Breit-Wigner distribution for the reconstructed on-shell $V$ with an effective $\Gamma_{V}=10 \mathrm{GeV}$. Middle: convolution of the partonic $q^{2}$ spectrum with a Breit-Wigner distribution for the reconstructed $h$ with an effective $\Gamma_{h}=10 \mathrm{GeV}$. Right: convolution with a Breit-Wigner for both $V$ and $h$ invariant masses. In all plots the color codes of the curves (and the corresponding normalization and parameter choice) is as in figure 1.

The effect of the experimental uncertainty is to suppress the peak in the threshold production region at low $q^{2}$. Introducing the effective smearing discussed above, the effect is not particularly pronounced, as shown in figure 2. A more accurate modeling of the experimental resolution would be needed in order to provide a more quantitative assessment of this effect, but this preliminary investigation indicates that the sensitivity to the Wilson coefficients is not significantly degraded by these concerns. Note that errors in the reconstruction of $q^{2}$ itself are mitigated by a coarse binning of the reconstructed spectrum.

\subsection{PDF effects, further distributions}

We produce the hadronic invariant mass distributions for $\sigma(p p \rightarrow Z h)$ by convoluting over MSTW2008 PDFs [44, 45]. Due to the typical enhancement of the threshold region in the presence of anomalous couplings, the utility of reconstructing the two body $d \sigma\left(q^{2}\right) / d q^{2}$ distribution is not significantly diminished by PDF effects. The effect of convoluting over the appropriate PDF's for the benchmark curves shown in figures 1-2 is illustrated in figure 3.

Deviations in $c_{1}$ when $c_{2}, c_{3} \sim 0$ only normalize the expected $\mathrm{SM}$ distribution (that is re-scaled by $c_{1}^{2}$ ). In this case, since no extra momentum dependence is present, the differential distributions of associated production offer no advantage over precise measurements of the associated production signal strengths. However, in the presence of BSM expressed through the effective couplings $c_{2,3}$, the non-SM momentum dependence in the form factor modifies the tail of the differential distributions substantially as shown in figure 3. Further, the threshold region is typically significantly enhanced. Even low statistics reconstructions of these spectra would offer the opportunity to significantly constrain anomalous interactions of a $0^{+}$state in the general EFT.

As shown in figure 3, the modification of the associated production cross section dependence on $q^{2}$ in the case of a nonzero $c_{2,3}$ is highly degenerate. This is why disentangling the effects of anomalous $c_{2,3}$ couplings would most likely require a combination of studies 

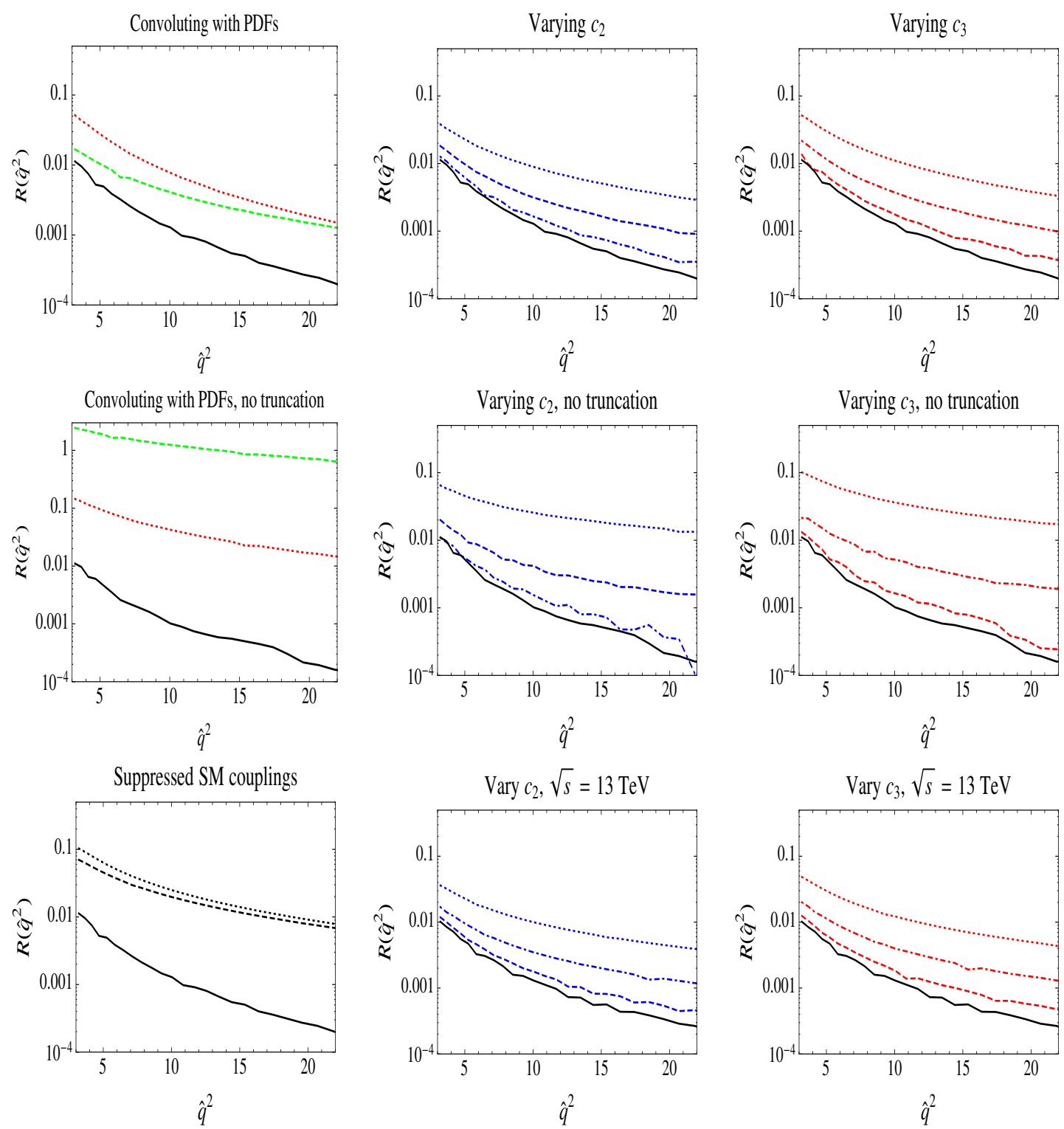

Figure 3. Differential $p p$ crossection normalized to the SM value: $R\left(\hat{q}^{2}\right)=\left[\sigma^{\mathrm{SM}}(p p \rightarrow Z h]^{-1} \times\right.$ $\times \mathrm{d} \sigma^{\mathrm{EFT}}(p p \rightarrow Z h) / \mathrm{d} \hat{q}^{2}$. Top Left: $p p$ cross section for the same $c_{i}$ used in figure $1(\sqrt{s}=8 \mathrm{TeV})$ using eq. (5.4). Top Middle: varying the parameter $c_{2}$ over values 0.01 (dashed), 0.05 (dotdashed) and 0.2 (dotted) using eq. (5.4). The parameters $c_{1}, c_{3}$ are fixed to 1,0 in this case. Top Right: varying the parameter $c_{3}$ over values 0.01 (dashed), 0.05 (dot-dashed) and 0.2 (dotted) using eq. (5.4). The parameters $c_{1}, c_{2}$ are fixed to 1,0 in this case. Middle row: same as the top row except the un-truncated expression for eq. (2.6) is used. Bottom Left: EFT parameters leading to a suppressed leading-order couplings of $h$ to the $Z$ : $c_{i}=(0.5,1,0.01)$ for the dashed curve and $c_{i}=(0.5,0.01,1)$ for the dotted curve. Bottom middle and right plot: same parameter choices as in the corresponding plots in the top row, for $\sqrt{s}=13 \mathrm{TeV}$.

of the effects in the associated production and in $h \rightarrow V \mathcal{F}$ decays. For illustrative purposes we also report the spectra corresponding to a suppressed leading-order couplings of $h$ to the $Z$, although this case is already ruled out by the present ATLAS [42] and CMS [43] 
results. The plots shown here should be interpreted as illustrative of how large the BSM effects can be in the associated production spectra, providing a motivation for experimental studies aimed at reconstructing these spectra from data.

\section{Conclusions}

In this paper we have examined the importance of using associated production spectra of the Higgs-like boson in constraining the properties of the newly discovered Boson $h$, that we have assumed to be a $0^{+}$state. Combining the results of this paper with past work [1], we have systematically developed a formalism to decompose and characterize the tree-point $h V \mathcal{F}$ Green's function, that can be probed both in $h$ decays $(h \rightarrow V \mathcal{F})$ and in $V h$ associated production $\left(p p \rightarrow \mathcal{F} \rightarrow h V\right.$ ). Given the experimental value of $m_{h}$, what can be probed in such processes is only the on-shell $h V \mathcal{F}$ Green's function, and not the $h V V$ one (that is kinematically forbidden). This implies that, in generic extensions of the $\mathrm{SM}$, it is necessary to incorporate a possible non-standard momentum dependence in the form factor decomposition of such processes. This non-SM $q^{2}$ dependence can make the differential spectra of these processes more sensitive probes of the nature of the Higgs-like Boson than just the total signal strength.

Our results indicate that associated production is a powerful probe of the non-SM $q^{2}$ dependence that could be present in the $h V \mathcal{F}$ Greens function, offering enhanced sensitivity to non-SM properties of the newly discovered state. This is simply due to the fact that, by construction, in this process $q^{2} / m_{V}^{2} \gg 1$ and the effect of a non-SM derivative expansion for effective operators involving the $h$ field is enhanced. We have also shown how, employing an EFT description of the form factors, low- $q^{2}$ and high- $q^{2}$ measurements of associated production constrain different combinations of the underlying parameters in the EFT construction. We have demonstrated how to map the $h V \mathcal{F}$ form factors into EFT approaches based on a linear or a non-linear realization of $\mathrm{SU}(2)_{\mathrm{L}} \times \mathrm{U}(1)_{\mathrm{Y}}$. This has allowed us to clarify the importance of $h \rightarrow V \mathcal{F}$ and $\mathcal{F} \rightarrow h V$ differential measurements as key probes to distinguish these two EFT approaches.

When a total signal strength is reported for associated production, we emphasize to the experimental collaborations that it is essential to report a corresponding average $\bar{q}^{2}$ in order for the underlying EFT parameters to be properly constrained. We also strongly encourage the experimental collaborations to report a reconstructed $d \sigma / d q^{2}$ spectrum for differing values of $q^{2}$ in associated production as soon as sufficient data is collected to allow this reconstruction. Even a low statistics reconstruction of this spectrum with a coarse binning is a key measurement to constraining the properties of the Higgs-like boson.

\section{Acknowledgments}

We thank Aneesh Manohar for useful discussions and collaboration on related material. We also thank Gian Giudice and Witold Skiba for useful discussions, and Jure Zupan for comments on the manuscript. This research was supported in part by the National Science 
Foundation under Grant No. PHY11-25915 at KITP Santa Barbara. G.I. acknowledges partial support by MIUR under project 2010YJ2NYW.

Open Access. This article is distributed under the terms of the Creative Commons Attribution License (CC-BY 4.0), which permits any use, distribution and reproduction in any medium, provided the original author(s) and source are credited.

\section{References}

[1] G. Isidori, A.V. Manohar and M. Trott, Probing the nature of the Higgs-like boson via $h \rightarrow V \mathcal{F}$ decays, Phys. Lett. B 728 (2014) 131 [arXiv:1305.0663] [InSPIRE].

[2] S. Bolognesi et al., On the spin and parity of a single-produced resonance at the LHC, Phys. Rev. D 86 (2012) 095031 [arXiv: 1208.4018] [INSPIRE].

[3] R. Boughezal, T.J. LeCompte and F. Petriello, Single-variable asymmetries for measuring the 'Higgs' boson spin and CP properties, arXiv:1208.4311 [INSPIRE].

[4] Y. Gao et al., Spin determination of single-produced resonances at hadron colliders, Phys. Rev. D 81 (2010) 075022 [arXiv:1001.3396] [INSPIRE].

[5] A. De Rujula, J. Lykken, M. Pierini, C. Rogan and M. Spiropulu, Higgs look-alikes at the LHC, Phys. Rev. D 82 (2010) 013003 [arXiv:1001.5300] [INSPIRE].

[6] S. Glashow, D.V. Nanopoulos and A. Yildiz, Associated production of Higgs bosons and Z particles, Phys. Rev. D 18 (1978) 1724 [INSPIRE].

[7] M. Ciccolini, S. Dittmaier and M. Krämer, Electroweak radiative corrections to associated WH and ZH production at hadron colliders, Phys. Rev. D 68 (2003) 073003 [hep-ph/0306234] [INSPIRE].

[8] A. Stange, W.J. Marciano and S. Willenbrock, Associated production of Higgs and weak bosons, with $H \rightarrow b \bar{b}$, at hadron colliders, Phys. Rev. D 50 (1994) 4491 [hep-ph/9404247] [INSPIRE].

[9] R. Kleiss, Z. Kunszt and W.J. Stirling, Discovering a light intermediate mass Higgs in proton proton collisions using associated production with $W$ bosons, Phys. Lett. B 253 (1991) 269 [INSPIRE].

[10] A. Belyaev et al., Technicolor walks at the LHC, Phys. Rev. D 79 (2009) 035006 [arXiv: 0809.0793] [INSPIRE].

[11] R. Frederix et al., $W$ and $Z / \gamma^{*}$ boson production in association with a bottom-antibottom pair, JHEP 09 (2011) 061 [arXiv:1106.6019] [INSPIRE].

[12] G. Ferrera, M. Grazzini and F. Tramontano, Associated WH production at hadron colliders: a fully exclusive QCD calculation at NNLO, Phys. Rev. Lett. 107 (2011) 152003 [arXiv: 1107.1164] [INSPIRE].

[13] J. Ellis, D.S. Hwang, V. Sanz and T. You, A fast track towards the 'Higgs' spin and parity, JHEP 11 (2012) 134 [arXiv:1208.6002] [INSPIRE].

[14] J. Ellis, V. Sanz and T. You, Associated production evidence against Higgs impostors and anomalous couplings, Eur. Phys. J. C 73 (2013) 2507 [arXiv: 1303.0208] [INSPIRE].

[15] E. Masso and V. Sanz, Limits on anomalous couplings of the Higgs to electroweak gauge bosons from LEP and LHC, Phys. Rev. D 87 (2013) 033001 [arXiv:1211.1320] [INSPIRE]. 
[16] S. Dawson, T. Han, W. Lai, A. Leibovich and I. Lewis, Resummation effects in vector-boson and Higgs associated production, Phys. Rev. D 86 (2012) 074007 [arXiv:1207.4207] [INSPIRE].

[17] C. Englert and M. McCullough, Modified Higgs sectors and NLO associated production, JHEP 07 (2013) 168 [arXiv: 1303.1526] [INSPIRE].

[18] A. Azatov and J. Galloway, Electroweak symmetry breaking and the Higgs boson: confronting theories at colliders, Int. J. Mod. Phys. A 28 (2013) 1330004 [arXiv:1212.1380] [InSPIRE].

[19] C. Grojean, E.E. Jenkins, A.V. Manohar and M. Trott, Renormalization group scaling of Higgs operators and $\Gamma(h->\gamma \gamma)$, JHEP 04 (2013) 016 [arXiv:1301.2588] [INSPIRE].

[20] R. Contino, M. Ghezzi, C. Grojean, M. Muhlleitner and M. Spira, Effective lagrangian for a light Higgs-like scalar, JHEP 07 (2013) 035 [arXiv: 1303.3876] [INSPIRE].

[21] P. Artoisenet et al., A framework for Higgs characterisation, JHEP 11 (2013) 043 [arXiv: 1306.6464] [INSPIRE].

[22] R. Alonso, M. Gavela, L. Merlo, S. Rigolin and J. Yepes, Flavor with a light dynamical "Higgs particle", Phys. Rev. D 87 (2013) 055019 [arXiv:1212.3307] [INSPIRE].

[23] J.R. Ellis, M.K. Gaillard and D.V. Nanopoulos, A phenomenological profile of the Higgs boson, Nucl. Phys. B 106 (1976) 292 [inSPIRE].

[24] B. Grinstein, C.W. Murphy and D. Pirtskhalava, Searching for new physics in the three-body decays of the Higgs-like particle, JHEP 10 (2013) 077 [arXiv: 1305.6938] [INSPIRE].

[25] E.E. Jenkins, A.V. Manohar and M. Trott, On gauge invariance and minimal coupling, JHEP 09 (2013) 063 [arXiv: 1305.0017] [INSPIRE].

[26] B. Grinstein and M. Trott, A Higgs-Higgs bound state due to new physics at a TeV, Phys. Rev. D 76 (2007) 073002 [arXiv:0704.1505] [INSPIRE].

[27] R. Contino, C. Grojean, M. Moretti, F. Piccinini and R. Rattazzi, Strong double Higgs production at the LHC, JHEP 05 (2010) 089 [arXiv: 1002.1011] [INSPIRE].

[28] C. Burgess, J. Matias and M. Pospelov, A Higgs or not a Higgs? What to do if you discover a new scalar particle, Int. J. Mod. Phys. A 17 (2002) 1841 [hep-ph/9912459] [INSPIRE].

[29] R.S. Chivukula and H. Georgi, Composite technicolor standard model, Phys. Lett. B 188 (1987) 99 [INSPIRE].

[30] L. Hall and L. Randall, Weak scale effective supersymmetry, Phys. Rev. Lett. 65 (1990) 2939 [INSPIRE].

[31] G. D'Ambrosio, G. Giudice, G. Isidori and A. Strumia, Minimal flavor violation: an effective field theory approach, Nucl. Phys. B 645 (2002) 155 [hep-ph/0207036] [INSPIRE].

[32] V. Cirigliano, B. Grinstein, G. Isidori and M.B. Wise, Minimal flavor violation in the lepton sector, Nucl. Phys. B 728 (2005) 121 [hep-ph/0507001] [INSPIRE].

[33] R. Alonso, M. Gavela, L. Merlo, S. Rigolin and J. Yepes, The effective chiral lagrangian for a light dynamical "Higgs particle", Phys. Lett. B 722 (2013) 330 [Erratum ibid. B 726 (2013) 926] [arXiv:1212.3305] [INSPIRE].

[34] G. Buchalla, O. Catà and C. Krause, Complete electroweak chiral lagrangian with a light Higgs at NLO, arXiv:1307.5017 [INSPIRE]. 
[35] G. Buchalla, O. Catà and C. Krause, On the power counting in effective field theories, arXiv:1312.5624 [INSPIRE].

[36] A. Manohar and H. Georgi, Chiral quarks and the nonrelativistic quark model, Nucl. Phys. B 234 (1984) 189 [INSPIRE].

[37] M. Soldate and R. Sundrum, Z couplings to pseudogoldstone bosons within extended technicolor, Nucl. Phys. B 340 (1990) 1 [InSPIRE].

[38] R.S. Chivukula, M.J. Dugan and M. Golden, Electroweak corrections in technicolor reconsidered, Phys. Lett. B 292 (1992) 435 [hep-ph/9207249] [INSPIRE].

[39] H. Georgi, Generalized dimensional analysis, Phys. Lett. B 298 (1993) 187 [hep-ph/9207278] [INSPIRE].

[40] B. Grzadkowski, M. Iskrzynski, M. Misiak and J. Rosiek, Dimension-six terms in the standard model lagrangian, JHEP 10 (2010) 085 [arXiv: 1008.4884] [INSPIRE].

[41] A. Pomarol, Invisibles seminar, http://invisibles.eu/journal-club (2013).

[42] ATLAS collaboration, Search for the standard model Higgs boson in produced in association with a vector boson and decaying to bottom quarks with the ATLAS detector,

ATLAS-CONF-2012-161 (2012).

[43] CMS collaboration, Search for the standard model higgs boson produced in association with $W$ or $Z$ bosons, and decaying to bottom quarks for LHCp 2013, CMS-PAS-HIG-13-012 (2013).

[44] A. Martin, W. Stirling, R. Thorne and G. Watt, Parton distributions for the LHC, Eur. Phys. J. C 63 (2009) 189 [arXiv:0901.0002] [InSPIRE].

[45] A. Martin, W. Stirling, R. Thorne and G. Watt, Uncertainties on $\alpha_{s}$ in global PDF analyses and implications for predicted hadronic cross sections, Eur. Phys. J. C 64 (2009) 653 [arXiv:0905.3531] [INSPIRE]. 